\title{
Approximate formulas for moderately small eikonal amplitudes
}

\author{
A.V. Kisselev* \\ Institute for High Energy Physics, NRC "Kurchatov Institute", \\ 142281 Protvino, Russian Federation
}

\begin{abstract}
The eikonal approximation for moderately small scattering amplitudes is considered. With the purpose of using for their numerical estimations, the formulas are derived which contain no Bessel functions, and, hence, no rapidly oscillating integrands. To obtain these formulas, the improper integrals of the first kind which contain products of the Bessel functions $J_{0}(z)$ are studied. The expression with four functions $J_{0}(z)$ is generalized. The expressions for the integrals with the product of five and six Bessel functions $J_{0}(z)$ are also found. The known formula for the improper integral with two functions $J_{\nu}(z)$ is generalized for non-integer $\nu$.
\end{abstract}

\section{Introduction}

The eikonal approximation was born in the study of a ray optics in which it is assumed that light travels in a straight line. This assumption works fine as long as the size of the obstacle $a$ is large compared to the wavelength of light $\lambda$.

In quantum mechanics the eikonal approximation works well for processes involving the scattering of particles with large incoming momentum $k$ and

*Electronic address: alexandre.kisselev@ihep.ru 
when the scattering angle $\theta$ is very small. In potential scattering the eikonal approximation may be used if [1], [2]

$$
E \gg V(\vec{r})
$$

where $E$ is the particle energy, and $V(\vec{r})$ is the interaction potential. The cross section is defined by the amplitude as

$$
\frac{d \sigma}{d \Omega}=\left|A\left(\vec{k}, \vec{k}^{\prime}\right)\right|^{2}
$$

where $\vec{k}$ and $\vec{k}^{\prime}$ are incoming and outgoing 3 -momenta of a particle. It is convenient to define a momentum transfer:

$$
t=-\left(\vec{k}-\vec{k}^{\prime}\right)^{2}=-2 k^{2}(1-\cos \theta) .
$$

The Born scattering amplitude looks like

$$
A_{B}\left(\vec{k}, \vec{k}^{\prime}\right)=-\frac{1}{4 \pi} \int d \vec{r} \exp \left[i\left(\vec{k}-\vec{k}^{\prime}\right) \vec{r}\right] V(\vec{r}) .
$$

In eikonal approximation for a spherically symmetric potential the amplitude can be presented in the form (see, for instance, [3]):

$$
A\left(\vec{k}, \vec{k}^{\prime}\right)=i k \int_{0}^{\infty} b d b J_{0}(b \sqrt{-t})\{1-\exp [i \chi(b)]\}
$$

with the eikonal $\chi(b)$ given by the formula

$$
\chi(b)=-\frac{k}{E} \int_{b}^{\infty} \frac{r d r}{\sqrt{r^{2}-b^{2}}} V(r) .
$$

Note that the eikonal is the Fourier transform of the Born amplitude,

$$
\chi(b)=\frac{1}{2 \pi k} \int d^{2} k \exp (-i \vec{k} \vec{b}) A_{B}\left(\vec{k}, \vec{k}^{\prime}\right)=\frac{1}{k} \int_{0}^{\infty} d t J_{0}(b \sqrt{-t}) A_{B}(s, t)
$$

where $s=k^{2}$. 
In perturbative quantum field theory an exponentiation in high-energy scattering processes similar to that of the Glauber approximation was studied in [4]. The eikonal representation can be derived in the framework of quasipotential approach [5] for small scattering angles and smooth quasipotentials [6]. In [7] an extention of the eikonal approach was developed which automatically takes into account off-shell unitarity.

The differential cross section is given by

$$
\frac{d \sigma(s, t)}{d t}=\frac{1}{16 \pi s^{2}}|A(s, t)|^{2}
$$

where $A(s, t)$ is the (dimensionless) scattering amplitude, with $s$ and $t=-q_{\perp}^{2}$ being Mandelstam variables. In the eikonal approximation the amplitude is defined by the formula

$$
A(s, t)=4 \pi i s \int_{0}^{\infty} d b b J_{0}(b \sqrt{-t})\{1-\exp [i \chi(s, b)]\}
$$

where $b$ is the impact parameter. In its turn, the eikonal $\chi(s, b)$ in (9) is related to the Born amplitude $A_{B}(s, t)$ by the Fourier-Bessel transformation,

$$
\chi(s, b)=\frac{1}{4 \pi s} \int_{0}^{\infty} d q_{\perp} q_{\perp} J_{0}\left(q_{\perp} b\right) A_{\mathrm{B}}\left(s,-q_{\perp}^{2}\right) .
$$

If $A_{\mathrm{B}}$ is approximated by reggeons, we come to so-called Regge-eikonal approach 1

Suppose, one needs to estimate the amplitude (and, correspondingly, the differential cross section) numerically at

$$
s \gg-t \gg m_{N}^{2}
$$

as, for example, it takes place in studying interaction of high-energy cosmic particles with atmospheric nucleons. As one can see from eqs. (9), (10), the amplitude is defined via $A_{\mathrm{B}}$ by iterated integral which contains rapidly oscillating Bessel functions. An attempt to calculate this integral in the kinematical region (11) with a high accuracy may face computing difficalties.

\footnotetext{
${ }^{1}$ In the presence of extra dimensions, the Born amplitude gets an additional contribution from gravi-reggeons (reggeized Kaluza-Klein gravitons in the $t$-channel) [8].
} 
The problem becomes much easier if $|\chi(s, b)| \ll 1$ for all $b$ at fixed $s$ under consideration. In such a case, one can use the expansion

$$
1-\exp (i \chi)=-i \chi+\mathrm{O}\left(\chi^{2}\right)
$$

and get well-known result $A(s, t) \simeq A_{\mathrm{B}}(s, t)$ (see eq. (21) below). However, there could be that $|\chi(s, b)|$ is moderately small (i.e. $|\chi(s, b)|<1$ ). In such a case, the use of expansion (12) is not enough to achieve a required accuracy of numerical calculations, and a few more terms must be kept in the r.h.s. of (12). This is the goal of the present paper.

\section{Moderately small eikonal amplitude}

In our analysis, we restrict ourselves to three terms in the Taylor series expansion of $\exp (i \chi)$ in eq. (9) 2

$$
1-\exp (i \chi)=-i\left(\chi-\frac{\chi^{3}}{6}\right)+\frac{\chi^{2}}{2}+\mathrm{O}\left(\chi^{4}\right) .
$$

Then the corresponding approximation for the amplitude looks like

$$
A(s, t) \simeq\left[A_{1}(s, t)-A_{3}(s, t)\right]+i A_{2}(s, t),
$$

where

$$
\begin{aligned}
& A_{1}(s, t)=4 \pi s \int_{0}^{\infty} d b b J_{0}(b \sqrt{-t}) \chi(s, b), \\
& A_{2}(s, t)=2 \pi s \int_{0}^{\infty} d b b J_{0}(b \sqrt{-t}) \chi^{2}(s, b), \\
& A_{3}(s, t)=\frac{2}{3} \pi s \int_{0}^{\infty} d b b J_{0}(b \sqrt{-t}) \chi^{3}(s, b) .
\end{aligned}
$$

Omitting terms of order $\mathrm{o}\left(\chi^{4}\right)$, we find

$$
\begin{aligned}
\frac{d \sigma(s, t)}{d t}=\frac{1}{16 \pi s^{2}} & \left\{\left|A_{1}(s, t)\right|^{2}-i\left[A_{1}(s, t) A_{2}^{*}(s, t)-A_{1}^{*}(s, t) A_{2}(s, t)\right]\right. \\
& +\left|A_{2}(s, t)\right|^{2}-\left[A_{1}(s, t) A_{3}^{*}(s, t)+A_{1}^{*}(s, t) A_{3}(s, t]\right\} .
\end{aligned}
$$

\footnotetext{
${ }^{2}$ For more than three terms in the r.h.s. of (13), see our comments in the end of this Section.
} 
In particular, if $A_{B}(s, t)$ has no imaginary part, then in the same approximation the differential cross section looks like

$$
\frac{d \sigma(s, t)}{d t}=\frac{1}{16 \pi s^{2}}\left\{A_{1}^{2}(s, t)+A_{2}^{2}(s, t)-2 A_{1}(s, t) A_{3}(s, t)\right\} .
$$

For a pure imaginary Born amplitude, $A_{1}$ and $A_{3}$ are pure imaginary, while $A_{2}$ is real, and we find:

$$
\begin{aligned}
\frac{d \sigma(s, t)}{d t}=\frac{1}{16 \pi s^{2}} & \left\{A_{1}^{2}(s, t)-2 i A_{1}(s, t) A_{2}(s, t)\right. \\
& \left.+A_{2}^{2}(s, t)+2 A_{1}(s, t) A_{3}(s, t)\right\} .
\end{aligned}
$$

Let us study three parts (15)-(17) of the amplitudes separately. Taking into account formulas (A.1), (A.2) presented in Appendix A, we obtain immediately from (15):

$$
\begin{aligned}
A_{1}(s, t) & =\int_{0}^{\infty} d b b J_{0}(b \sqrt{-t}) \int_{0}^{\infty} d q_{\perp} q_{\perp} J_{0}\left(q_{\perp} b\right) A_{B}\left(s, q_{\perp}\right) \\
& =\int_{0}^{\infty} d q_{\perp} q_{\perp} A_{B}\left(s, q_{\perp}\right) \int_{0}^{\infty} d b b J_{0}\left(q_{\perp} b\right) J_{0}(b \sqrt{-t})=A_{B}(s, \sqrt{-t}) .
\end{aligned}
$$

The second part of the amplitude (16) can be analyzed by the use of eqs. (A.12), (A.13) from Appendix A. As a result, we find:

$$
\begin{aligned}
& A_{2}(s, t) \\
& =\frac{1}{8 \pi s} \int_{0}^{\infty} d b b J_{0}(b \sqrt{-t}) \int_{0}^{\infty} d q_{\perp} q_{\perp} J_{0}\left(q_{\perp} b\right) A_{B}\left(s, q_{\perp}\right) \int_{0}^{\infty} d q_{\perp}^{\prime} q_{\perp}^{\prime} J_{0}\left(q_{\perp}^{\prime} b\right) A_{B}\left(s, q_{\perp}^{\prime}\right) \\
& =\frac{1}{8 \pi s} \int_{0}^{\infty} d q_{\perp} q_{\perp} A_{B}\left(s, q_{\perp}\right) \int_{0}^{\infty} d q_{\perp}^{\prime} q_{\perp}^{\prime} A_{B}\left(s, q_{\perp}^{\prime}\right) \int_{0}^{\infty} d b b J_{0}\left(q_{\perp} b\right) J_{0}\left(q_{\perp}^{\prime} b\right) J_{0}(b \sqrt{-t}) \\
& =\frac{1}{4 \pi^{2} s} \int_{0}^{\infty} d q_{\perp} q_{\perp} A_{B}\left(s, q_{\perp}\right) \int_{0}^{\infty} d q_{\perp}^{\prime} q_{\perp}^{\prime} A_{B}\left(s, q_{\perp}^{\prime}\right) \theta\left(\sqrt{-t}-\left|q_{\perp}-q_{\perp}^{\prime}\right|\right) \\
& \times \theta\left(q_{\perp}+q_{\perp}^{\prime}-\sqrt{-t}\right) \frac{1}{\sqrt{\left[-t-\left(q_{\perp}-q_{\perp}^{\prime}\right)^{2}\right]\left[\left(q_{\perp}+q_{\perp}^{\prime}\right)^{2}+t\right]}} .
\end{aligned}
$$


Let us define new variables

$$
q_{1}=q_{\perp}+q_{\perp}^{\prime}, \quad q_{2}=q_{\perp}-q_{\perp}^{\prime} .
$$

In terms of them $A_{2}(s, t)$ can be rewritten in the form:

$$
\begin{aligned}
& A_{2}(s, t)=\frac{1}{32 \pi^{2} s} \int_{0}^{\infty} d q_{1} \int_{-q_{1}}^{q_{1}} d q_{2} A_{B}\left[s,\left(q_{1}+q_{2}\right) / 2\right] A_{B}\left[s,\left(q_{1}-q_{2}\right) / 2\right] \\
& \times \theta\left(q_{1}-\sqrt{-t}\right) \theta\left(\sqrt{-t}-\left|q_{2}\right|\right) \frac{\left(q_{1}^{2}-q_{2}^{2}\right)}{\sqrt{\left(-t-q_{2}^{2}\right)\left(q_{1}^{2}+t\right)}} \\
& =\frac{1}{16 \pi^{2} s} \int_{\sqrt{-t}}^{\infty} d q_{1} \int_{0}^{\sqrt{-t}} d q_{2} A_{B}\left[s,\left(q_{1}+q_{2}\right) / 2\right] A_{B}\left[s,\left(q_{1}-q_{2}\right) / 2\right] \\
& \times \frac{\left(q_{1}^{2}-q_{2}^{2}\right)}{\sqrt{\left(-t-q_{2}^{2}\right)\left(q_{1}^{2}+t\right)}} .
\end{aligned}
$$

After change of variables $q_{i}=x_{i} \sqrt{-t}(i=1,2)$ we come to the equation

$$
\begin{aligned}
A_{2}(s, t) & =\frac{1}{16 \pi^{2}}\left(\frac{-t}{s}\right) \int_{1}^{\infty} d x_{1} \int_{0}^{1} d x_{2} \frac{\left(x_{1}^{2}-x_{2}^{2}\right)}{\sqrt{\left(x_{1}^{2}-1\right)\left(1-x_{2}^{2}\right)}} \\
& \times A_{B}\left[s, \sqrt{-t}\left(x_{1}+x_{2}\right) / 2\right] A_{B}\left[s, \sqrt{-t}\left(x_{1}-x_{2}\right) / 2\right] .
\end{aligned}
$$

For the third part of the amplitude (17) we get

$$
\begin{aligned}
A_{3}(s, t) & =\frac{1}{96 \pi^{2} s^{2}} \int_{0}^{\infty} d b b J_{0}(b \sqrt{-t}) \int_{0}^{\infty} d q_{\perp} q_{\perp} J_{0}\left(q_{\perp} b\right) A_{B}\left(s, q_{\perp}\right) \\
& \times \int_{0}^{\infty} d q_{\perp}^{\prime} q_{\perp}^{\prime} J_{0}\left(q_{\perp}^{\prime} b\right) A_{B}\left(s, q_{\perp}^{\prime}\right) \int_{0}^{\infty} d q_{\perp}^{\prime \prime} q_{\perp}^{\prime \prime} J_{0}\left(q_{\perp}^{\prime \prime} b\right) A_{B}\left(s, q_{\perp}^{\prime \prime}\right) \\
& =\frac{1}{96 \pi^{2} s^{2}} \int_{0}^{\infty} d q_{\perp} q_{\perp} A_{B}\left(s, q_{\perp}\right) \int_{0}^{\infty} d q_{\perp}^{\prime} q_{\perp}^{\prime} A_{B}\left(s, q_{\perp}^{\prime}\right) \int_{0}^{\infty} d q_{\perp}^{\prime \prime} q_{\perp}^{\prime \prime} A_{B}\left(s, q_{\perp}^{\prime \prime}\right) \\
& \times \int_{0}^{\infty} d b b J_{0}\left(q_{\perp} b\right) J_{0}\left(q_{\perp}^{\prime} b\right) J_{0}\left(q_{\perp}^{\prime \prime} b\right) J_{0}(b \sqrt{-t}) .
\end{aligned}
$$


By using eqs. (A.21), (A.44) from Appendix A, the amplitude $A_{3}(s, t)$ (26) can be be presented in the form:

$$
\begin{aligned}
A_{3}(s, t) & =\frac{1}{96 \pi^{2}}\left(\frac{-t}{s}\right)^{2} \int_{0}^{\infty} d x A_{B}(s, \sqrt{-t} x) \int_{0}^{\infty} d x^{\prime} A_{B}\left(s, \sqrt{-t} x^{\prime}\right) \\
& \times \int_{0}^{\infty} d x^{\prime \prime} A_{B}\left(s, \sqrt{-t} x^{\prime \prime}\right) G\left(x, x^{\prime}, x^{\prime \prime}\right) .
\end{aligned}
$$

Here we introduced the function

$$
G\left(x, x^{\prime}, x^{\prime \prime}\right)=\left\{\begin{array}{cc}
\frac{1}{\pi^{2} A} K\left(\frac{\sqrt{B}}{A}\right), & A^{2}>B, \\
\frac{1}{\pi^{2} \sqrt{B}} K\left(\frac{A}{\sqrt{B}}\right), & 0 \leqslant A^{2}<B, \\
0, & A^{2}<0,
\end{array}\right.
$$

with $K(k)$ being the complete elliptic integral of the first kind (A.24) and notations

$$
16 A^{2}=\left[\left(x^{\prime \prime}+1\right)^{2}-\left(x-x^{\prime}\right)^{2}\right]\left[\left(x+x^{\prime}\right)^{2}-\left(x^{\prime \prime}-1\right)^{2}\right], \quad B=x x^{\prime} x^{\prime \prime} .
$$

Let us define new variables

$$
x_{1}=x+x^{\prime}, \quad x_{2}=x-x^{\prime}, \quad x_{3}=x^{\prime \prime},
$$

They run in the region restricted by the following inequalities

$$
0 \leqslant x_{1} \leqslant \infty, \quad-x_{1} \leqslant x_{2} \leqslant x_{1}, \quad 0 \leqslant x_{3} \leqslant \infty
$$

and

$$
\left[\left(x_{3}+1\right)^{2}-x_{2}^{2}\right]\left[x_{1}^{2}-\left(x_{3}-1\right)^{2}\right]>0 .
$$

Then we find from eqs. (27)-(32):

$$
\begin{aligned}
A_{3}(s, t) & =\frac{1}{96 \pi^{2}}\left(\frac{-t}{s}\right)^{2} \int_{0}^{\infty} d x_{1} \int_{-x_{1}}^{x_{1}} d x_{2} \int_{0}^{\infty} d x_{3} \theta\left(\left[\left(x_{3}+1\right)^{2}-x_{2}^{2}\right]\left[x_{1}^{2}-\left(x_{3}-1\right)^{2}\right]\right) \\
& \times H\left(s, t ; x_{1}, x_{2}, x_{3}\right)
\end{aligned}
$$


where

$$
\begin{aligned}
H\left(s, t ; x_{1}, x_{2}, x_{3}\right) & =A_{B}\left(s, \sqrt{-t}\left(x_{1}+x_{2}\right) / 2\right) A_{B}\left(s, \sqrt{-t}\left(x_{1}-x_{2}\right) / 2\right) \\
& \times A_{B}\left(s, \sqrt{-t} x_{3}\right) G\left(\left(x_{1}+x_{2}\right) / 2,\left(x_{1}-x_{2}\right) / 2, x_{3}\right) .
\end{aligned}
$$

By using formula from Appendix B, we get

$$
\begin{aligned}
A_{3}(s, t) & =\frac{1}{96 \pi^{2}}\left(\frac{-t}{s}\right)^{2}\left[\int_{0}^{1} d x_{1} \int_{0}^{x_{1}} d x_{2} \int_{1-x_{1}}^{x_{1}+1} d x_{3}+\int_{1}^{2} d x_{1} \int_{0}^{1} d x_{2} \int_{0}^{x_{1}+1} d x_{3}\right. \\
& \left.+\int_{1}^{2} d x_{1} \int_{1}^{x_{1}} d x_{2} \int_{\left|x_{2}\right|-1}^{x_{1}+1} d x_{3}+\int_{2}^{\infty} d x_{1} \int_{0}^{x_{1}+1} d x_{2} \int_{0}^{x_{1}} d x_{3}+\int_{2}^{x_{1}+1} d x_{1} \int_{1} d x_{2} \int_{\left|x_{2}\right|-1} d x_{3}\right] \\
& \times\left[H\left(s, t ; x_{1}, x_{2}, x_{3}\right)+H\left(s, t ; x_{1},-x_{2}, x_{3}\right)\right] .
\end{aligned}
$$

Our formulas (21), (25), (35) and (18) can be applied to numerical calculations of differential cross sections at given fixed $s$ and $t$, provided that the Born amplitude $A_{B}(s, t)$ is known and approximation (13) is justified. The advantage of these integrals lies in the fact that they contain no rapidly oscillating integrands 3 Of course, we assume that the Born amplitude has no such oscillations in variable $t$.

If more terms should be kept in expansion (13), integrals with more than four Bessel functions $J_{0}(z)$ have to be used. The corresponding formulas are presented in Appendix A (see eqs. (A.52), (A.57)).

\section{Acknowledgements}

The author is indebted to V.A. Petrov and V.E. Rochev for valuable remarks.

\section{Appendix A}

In this section we consider improper integrals of the first kind which contain products of the Bessel functions. The well-known formula for the integral with two functions $J_{\nu}(z)$ is generalized for non-integer $\nu$. The expression

\footnotetext{
${ }^{3}$ The complete elliptic integral of the first kind $K(k)$ in (28) is a monotone increasing function of its modulus $k(0 \leqslant k<1)$.
} 
for the tabulated integral with four functions $J_{0}(z)$ is defined more exactly. Finally, the analytic expression for the integrals with the product of five and six Bessel functions $J_{0}(z)$ are derived.

Let us start from the integral with two Bessel functions $J_{n}(z)$ :

$$
F_{2}^{(n)}(a, b)=\int_{0}^{\infty} d x x J_{n}(a x) J_{n}(b x), \quad n=0,1, \ldots
$$

For $a, b>0$, it is known to be (see eq. (3.108) in [9], as well as eq. 6.512.8. in [10])

$$
F_{2}^{(n)}(a, b)=\frac{1}{a} \delta(a-b) .
$$

Let us calculate more general integral containing Bessel functions with non-iteger index $\nu$ (see the problem 3.16(a) in ref. [9]),

$$
F_{2}^{(\nu)}(a, b)=\int_{0}^{\infty} d x x J_{\nu}(a x) J_{\nu}(b x)
$$

We start from the Weber's second exponential integral (see, for instance, eq. 13.31.(1) in [11]):

$$
\begin{aligned}
I_{W}(a, b ; p) & =\int_{0}^{\infty} d x x \exp \left(-p^{2} x^{2}\right) J_{\nu}(a x) J_{\nu}(b x) \\
& =\frac{1}{2 p^{2}} \exp \left[-\left(a^{2}+b^{2}\right) / 4 p^{2}\right] I_{0}\left(\frac{a b}{2 p^{2}}\right),
\end{aligned}
$$

where $I_{\nu}(z)$ is the modified Bessel function of the first kind. This formula is valid for $|\arg p|<\pi / 4$, Re $\nu>-1$. The parameters $a$ and $b$ can be arbitrary complex numbers.

Let $p>0$ in what follows. For $|\arg a b|<\pi / 2$ one can apply the asymptotic formula 7.23.(2) from [11]:

$$
\left.I_{\nu}\left(\frac{a b}{2 p^{2}}\right)\right|_{p \rightarrow 0}=\frac{1}{\sqrt{\pi a b / p^{2}}} \exp \left(a b / 2 p^{2}\right)\left[1+\mathrm{O}\left(p^{2}\right)\right]
$$

and obtain

$$
\left.I_{W}(a, b ; p)\right|_{p \rightarrow 0}=\frac{1}{\sqrt{a b}} \frac{1}{2 \sqrt{\pi p^{2}}} \exp \left(-(a-b)^{2} / 4 p^{2}\right)\left[1+\mathrm{O}\left(p^{2}\right)\right] .
$$


It is known that [12] $(t>0)$

$$
\left.\frac{1}{2 \sqrt{\pi t}} \exp \left(-x^{2} / 4 t\right)\right|_{t \rightarrow 0} \rightarrow \delta(x)
$$

As a result, we come to a generalization of the formula (A.2) for $\operatorname{Re} \nu>-1$, $|\arg a b|<\pi / 2$ :

$$
F_{2}^{(\nu)}(a, b)=\left.I_{W}(a, b ; p)\right|_{p \rightarrow 0}=\frac{1}{\sqrt{a b}} \delta(a-b) .
$$

For $\operatorname{Re} \nu>-1$ but positive $a$ and $b$, formula (A.8) can be also derived by using one of the discontinuous Weber-Schafheitlin integrals (see eq. 11.4.41. in [13], or 6.575.1 in [10] and eq. 2.12.31.1. in [14]):

$$
\int_{0}^{\infty} d x x^{\mu-\nu+1} J_{\mu}(a x) J_{\nu}(b x)=\left\{\begin{array}{cl}
0, & 0<b<a, \\
\frac{2^{\mu-\nu+1} a^{\mu}\left(b^{2}-a^{2}\right)^{\nu-\mu-1}}{b^{\nu} \Gamma(\nu-\mu)}, & b>a>0,
\end{array}\right.
$$

where $\operatorname{Re} \nu>\operatorname{Re} \mu>-1$. Let us put $\mu=\nu-(1+\lambda)$ in (A.9), and take the limit $\lambda \rightarrow-1(\lambda+1>0)$. Then we find for $a, b>0, \operatorname{Re} \nu>-1$ :

$$
\begin{aligned}
\int_{0}^{\infty} d x x J_{\nu}(a x) J_{\nu}(b x) & =\lim _{\mu \rightarrow \nu} \int_{0}^{\infty} d x x^{\mu-\nu+1} J_{\mu}(a x) J_{\nu}(b x) \\
& =\left(\frac{a}{b}\right)^{\nu} \lim _{\lambda \rightarrow-1} \frac{2^{-\lambda}}{a^{1+\lambda}} \frac{\left(b^{2}-a^{2}\right)_{+}^{\lambda}}{\Gamma(1+\lambda)}=\frac{1}{a} \delta(a-b)
\end{aligned}
$$

where we used the formula [12]

$$
\lim _{\lambda=-1} \frac{x_{+}^{\lambda}}{\Gamma(\lambda+1)}=\delta(x),
$$

The integral with three Bessel functions $J_{0}(z)$,

$$
F_{3}(a, b, c)=\int_{0}^{\infty} d x x J_{0}(a x) J_{0}(b x) J_{0}(c x),
$$


where $a, b, c>0$, is given by the formula (see, for instance, eq. 13.46(3) in [11], or eqs. 2.12.42.14. and eq. 2.12.42.15. in [14]):

$$
F_{3}(a, b, c)=\left\{\begin{array}{cc}
\frac{1}{2 \pi \Delta_{3}}, & \Delta_{3}^{2}>0 \\
0, & \Delta_{3}^{2}<0
\end{array}\right.
$$

where

$$
16 \Delta_{3}^{2}=\left[c^{2}-(a-b)^{2}\right]\left[(a+b)^{2}-c^{2}\right],
$$

Note that the integral in (A.13) is divergent if $\Delta_{3}^{2}=0$ (it takes place when one of the parameters is equal to the sum of the others, say, $c=a+b>0$ ).

Is eq. (A.13) in agreement with eq. (A.2)? To estimate expression (A.13) in the limit $c=0$ (but $a, b>0$ ), let us put $c=\varepsilon \ll 1$. We have

$$
\left.\frac{1}{2 \pi \Delta_{3}}\right|_{c=\varepsilon \ll 1} \simeq \frac{2}{(a+b)} \frac{1}{\pi \sqrt{\varepsilon^{2}-(a-b)^{2}}} .
$$

Consider the function

$$
f_{\varepsilon}(x)=\theta(\varepsilon+x) \theta(\varepsilon-x) \frac{1}{\pi \sqrt{\varepsilon^{2}-x^{2}}} .
$$

We find from (A.16) that

$$
\lim _{\varepsilon \rightarrow 0} \int_{\alpha}^{\beta} d x f_{\varepsilon}(x)=\left\{\begin{array}{c}
0, \quad \alpha<\beta<0 \text { or } 0<\alpha<\beta, \\
1, \quad \alpha<0<\beta .
\end{array}\right.
$$

Let us show that $f_{\varepsilon}(x)$ is a delta-like sequence. Consider the following sequence of antiderivative functions

$$
F_{\varepsilon}(x)=\int_{-1}^{x} f_{\varepsilon}(z) d z
$$

In the limit $\varepsilon \rightarrow 0$ the function $F_{\varepsilon}(x)$ tends to a constant value which is equal to zero at $x<0$ and to 1 at $x>0$. At the same time, it is uniformly bounded in $\varepsilon$ within each interval. Consequently,

$$
\left.F_{\varepsilon}(x)\right|_{\varepsilon \rightarrow 0}=\theta(x)
$$


and, correspondingly,

$$
\left.f_{\varepsilon}(x)\right|_{\varepsilon \rightarrow 0}=\theta^{\prime}(x)=\delta(x) .
$$

As a result, we reproduce eq. (A.2) (for $n=0$ ) from eq. (A.13) in the limit $c=0$.

Consider the integral with four Bessel functions $J_{0}(z)$ :

$$
F_{4}(a, b, c, d)=\int_{0}^{\infty} d x x J_{0}(a x) J_{0}(b x) J_{0}(c x) J_{0}(d x),
$$

with $a, b, c, d>0$. In [14] (see eq. 2.12.44.1.) the following formula is presented:

$$
F_{4}(a, b, c, d)=\left\{\begin{array}{cc}
\frac{1}{\pi^{2} \Delta_{4}} K\left(\frac{\sqrt{a b c d}}{\Delta_{4}}\right), & \Delta_{4}^{2}>a b c d, \\
\frac{1}{\pi^{2} \sqrt{a b c d}} K\left(\frac{\Delta_{4}}{\sqrt{a b c d}}\right), & \Delta_{4}^{2}<a b c d,
\end{array}\right.
$$

where

$$
16 \Delta_{4}^{2}=(a+b+c-d)(a+b+d-c)(a+c+d-b)(b+c+d-a),
$$

and

$$
K(k)=\int_{0}^{\pi / 2} \frac{d \theta}{\sqrt{1-k^{2} \sin ^{2} \theta}}=\int_{0}^{1} \frac{d t}{\sqrt{\left(1-t^{2}\right)\left(1-k^{2} t^{2}\right)}}
$$

is the complete elliptic integral of the first kind $(0 \leqslant k<1)$ [15]. For $\Delta_{4}^{2}=a b c d$ the integral (A.21) is not defined. Indeed, the function $K(k)$ has the branch point $k=1$, and it diverges at $k \rightarrow 1$ as [15]

$$
\left.K(k)\right|_{k \rightarrow 1} \simeq \ln \left(4 / \sqrt{1-k^{2}}\right) .
$$

Unfortunately, eq. (A.22) does not cover one important case $\Delta_{4}^{2}<0.4$ Let us analyze this case in detail and show that $F_{4}(a, b, c, d)=0$ for $\Delta_{4}^{2}<0$.

\footnotetext{
${ }^{4}$ If one applies the expression in the second line of eq. (A.22) to negative $\Delta_{4}^{2}$, he gives a wrong result.
} 
According to formula 2.12.44.7. from [14],

$$
\begin{aligned}
A_{\alpha}^{(n)}\left(c_{1}, \ldots, c_{n}\right) & =\int_{0}^{\infty} d x x^{\alpha-1} \prod_{k=1}^{n} J_{0}\left(c_{k} x\right)=\frac{2^{\alpha-1}}{c_{n}^{\alpha}} \frac{\Gamma(\alpha / 2)}{\Gamma(1-\alpha / 2)} \\
& \times F_{C}^{(n-1)}(\frac{\alpha}{2}, \frac{\alpha}{2} ; \underbrace{1, \ldots 1}_{n-1} ; \frac{c_{1}^{2}}{c_{n}^{2}}, \ldots \frac{c_{n-1}^{2}}{c_{n}^{2}}),
\end{aligned}
$$

for $0<\operatorname{Re} \alpha<n / 2+1 ; c_{k}>0, k=1, \ldots n ; c_{n}>c_{1}+\ldots+c_{n-1}$. Here

$$
\begin{aligned}
& F_{C}^{(n)}\left(a, b ; c_{1}, \ldots c_{n} ; z_{1}, \ldots z_{n}\right) \\
& =\sum_{k_{1}, \ldots k_{n}=0}^{\infty} \frac{(a)_{k_{1}+\ldots k_{n}}(b)_{k_{1}+\ldots k_{n}}}{\left(c_{1}\right)_{k_{1}} \ldots\left(c_{n}\right)_{k_{n}}} \frac{z_{1}^{k_{1}} \ldots z_{n}^{k_{n}}}{k_{1} ! \ldots k_{n} !}
\end{aligned}
$$

is the Lauricella function, $(a)_{k}=\Gamma(a+k) / \Gamma(a)$ being the Pochhammer symbol. The series in (A.27) converges if

$$
\sum_{i=1}^{n} \sqrt{\left|z_{i}\right|}<1 .
$$

It follows from (A.26) that $A_{2}^{(4)}\left(c_{1}, \ldots, c_{4}\right)=0$ for $c_{1}, \ldots c_{4}>0, c_{4}>c_{1}+$ $c_{2}+c_{3}$.

We need to analyze all possible relations between the parameters $a, d, c, d$. If all of them all equal, $a=b=c=d$, we get $\Delta_{4}^{2}=a^{4}=a b c d$. If they are pairwise equal, say, $a=b, c=d$, but $a \neq c$, we find again that $\Delta_{4}^{2}=a^{2} c^{2}=$ $a b c d$. In both cases the integral $F_{4}(a, b, c, d)$ does not exists (see comments after eq. (A.24) $)$.

If $a=b=c \neq d$, the inequality $\Delta_{4}^{2}<0$ is realized only for $d>3 a$ (i.e. for $d>a+b+c)$. In the case when only two parameters are equal, say, $a=b \neq c$, $a \neq d, c \neq d$, the inequality $\Delta_{4}^{2}<0$ holds if $\max (c, d)>2 a+\min (c, d)$ (for instance, $d>a+b+c$, if $d>c$ ).

Finally all the parameters can be different. Let, for example, $d$ be the largest one, $d>a, b, c$. If $d<a+b+c$, then $\Delta_{4}^{2}>0$, and we come to expressions (A.22). Otherwise, if $d>a+b+c$, then $\Delta_{4}^{2}<0$. Putting $n=4$, $\alpha=2, c_{1}=a, c_{2}=b, c_{3}=c$ and $c_{4}=d$ in eq. (A.26), and taking into account that $\left.\Gamma(1-\alpha / 2)\right|_{\alpha \rightarrow 2} \rightarrow 2 /(2-\alpha)$, we conclude from all said above that

$$
F_{4}(a, b, c, d)=A_{2}^{(4)}(a, b, c, d)=0, \quad \Delta_{4}^{2}<0 .
$$


The condition (A.29) can be derived in a different way. Let us use the integral representation for the Bessel functions [16]:

$$
J_{n}(z)=\frac{1}{2 \pi} \int_{0}^{2 \pi} d \varphi \exp [i(z \sin \varphi-n \varphi)], \quad n=0,1,2 \ldots
$$

Then we get

$$
\begin{aligned}
F_{4}(a, b, c, d) & =\frac{1}{(2 \pi)^{4}} \int_{0}^{\infty} B d B \int_{0}^{2 \pi} d \varphi_{1} \int_{0}^{2 \pi} d \varphi_{2} \int_{0}^{2 \pi} d \varphi_{3} \int_{0}^{2 \pi} d \varphi_{4} \\
& \times \exp \left(i B\left(a \sin \varphi_{1}+b \sin \varphi_{2}+c \sin \varphi_{3}+d \sin \varphi_{4}\right)\right. \\
& =\frac{1}{(2 \pi)^{5}} \int_{0}^{2 \pi} d \varphi_{1} \int_{0}^{2 \pi} d \varphi_{2} \int_{0}^{2 \pi} d \varphi_{3} \int_{0}^{2 \pi} d \varphi_{4} \int_{0}^{\infty} d \vec{B} \\
& \times \exp [i \vec{B}(\vec{a}+\vec{b}+\vec{c}+\vec{d})]
\end{aligned}
$$

where

$$
\vec{B}=(0, B),
$$

and two-dimensional vectors are introduced:

$$
\begin{aligned}
& \vec{a}=\left(a \cos \varphi_{1}, a \sin \varphi_{1}\right), \\
& \vec{b}=\left(b \cos \varphi_{2}, b \sin \varphi_{2}\right), \\
& \vec{c}=\left(c \cos \varphi_{3}, c \sin \varphi_{3}\right), \\
& \vec{d}=\left(d \cos \varphi_{4}, d \sin \varphi_{4}\right) .
\end{aligned}
$$

Since

$$
\begin{aligned}
& \frac{1}{(2 \pi)^{2}} \int_{0}^{\infty} d \vec{B} \exp [i \vec{B}(\vec{a}+\vec{b}+\vec{c}+\vec{d})]=\delta^{(2)}(\vec{a}+\vec{b}+\vec{c}+\vec{d}) \\
& =\delta\left(a \sin \varphi_{1}+b \sin \varphi_{2}+c \sin \varphi_{3}+d \sin \varphi_{4}\right) \\
& \times \delta\left(a \cos \varphi_{1}+b \cos \varphi_{2}+c \cos \varphi_{3}+d \cos \varphi_{4}\right)
\end{aligned}
$$

\footnotetext{
${ }^{5}$ This representation was obtained by F.W. Bessel.
} 
we find that

$$
\begin{aligned}
F_{4}(a, b, c, d) & =\frac{1}{(2 \pi)^{3}} \int_{0}^{2 \pi} d \varphi_{1} \int_{0}^{2 \pi} d \varphi_{2} \int_{0}^{2 \pi} d \varphi_{3} \int_{0}^{2 \pi} d \varphi_{4} \\
& \times \delta\left(a \sin \varphi_{1}+b \sin \varphi_{2}+c \sin \varphi_{3}+d \sin \varphi_{4}\right) \\
& \times \delta\left(a \cos \varphi_{1}+b \cos \varphi_{2}+c \cos \varphi_{3}+d \cos \varphi_{4}\right) .
\end{aligned}
$$

As it follows from the consideration presented above, $\Delta_{4}^{2}<0$ is realized if only one of the parameters $a, b, c, d$ is larger than the sum of all other ones. Without loss of generality, let us assume that $d>a+b+c$. The integral (A.35) is non-zero if only

$$
\begin{aligned}
a \sin \varphi_{1}+b \sin \varphi_{2}+c \sin \varphi_{3}+d \sin \varphi_{4} & =0 \\
a \cos \varphi_{1}+b \cos \varphi_{2}+c \cos \varphi_{3}+d \cos \varphi_{4} & =0
\end{aligned}
$$

In particular, in means that the following equation

$$
\begin{aligned}
1 & =\sin ^{2} \varphi_{4}+\cos ^{2} \varphi_{4}=\frac{1}{d^{2}}\left[a^{2}+b^{2}+c^{2}+2 a b \cos \left(\varphi_{1}-\varphi_{2}\right)\right. \\
& \left.+2 b c \cos \left(\varphi_{2}-\varphi_{3}\right)+2 c a \cos \left(\varphi_{3}-\varphi_{1}\right)\right]=A(a, b, c)
\end{aligned}
$$

should be valid. But the evident bound,

$$
A(a, b, c) \leqslant \frac{(a+b+c)^{2}}{d^{2}}<1
$$

says us that the equations (A.36) cannot be simultaneously satisfied, and we come again to the condition (A.29) 6

It is necessary to verify whether the integral $F_{4}(a, b, c, d)$ (A.21) exists for $\Delta_{4}^{2}=0$ (inequalities $a, b, c, d>0$ are assumed). Note that the equality $\Delta_{4}^{2}=0$ holds only if one of the parameters $a, b, c, d$ is equal to the sum of other three parameters. Let $d=a+b+c$. The asymptotic formula for the Bessel function [16],

$$
\left.J_{0}(z)\right|_{|z| \gg 1}=\sqrt{\frac{2}{\pi z}} \cos \left(z-\frac{\pi}{4}\right)\left[1+\mathrm{O}\left(|z|^{-1}\right)\right], \quad|\arg z|<\pi,
$$

\footnotetext{
${ }^{6}$ In the same way, one can show that $F_{3}(a, b, c)=0$ if $\Delta_{3}^{2}<0$.
} 
can be exploited, that results in the following expression $(x>0)$

$$
\begin{aligned}
& \left.J_{0}(a x) J_{0}(b x) J_{0}(c x) J_{0}[(a+b+c) x]\right|_{x \gg 1}=\frac{1}{2 \pi^{2} x^{2} \sqrt{a b c(a+b+c)}} \\
& \times\{\cos (2 a x)+\cos (2 b x)+\cos (2 c x) \\
& \quad+\sin [2(a+b) x]+\sin [2(b+c) x]+\sin [2(a+c) x]\}\left[1+\mathrm{O}\left(x^{-1}\right)\right] \\
& =J_{\text {asym }}(x)+\mathrm{O}\left(x^{-3}\right) .
\end{aligned}
$$

Then we can write for some fixed $x_{0}>0$ :

$$
\begin{aligned}
\left.F_{4}(a, b, c, d)\right|_{d=a+b+c} & =\int_{0}^{x_{0}} d x x J_{0}(a x) J_{0}(b x) J_{0}(c x) J_{0}[(a+b+c) x] \\
& +\int_{x_{0}}^{\infty} d x x\left\{J_{0}(a x) J_{0}(b x) J_{0}(c x) J_{0}[(a+b+c) x]-J_{a s y m}(x)\right\} \\
& +\int_{x_{0}}^{\infty} d x x J_{\text {asym }}(x),
\end{aligned}
$$

The first integral in (A.41) is obviously well-defined. The integrand in the second integral decreases rapidly at $x \rightarrow \infty$ as $\mathrm{O}\left(x^{-2}\right)$. The third integral in (A.41) is a linear combination of sine integrals

$$
\operatorname{si}(z)=-\int_{z}^{\infty} \frac{\sin t}{t} d t
$$

with $z=2(a+b) x_{0}, 2(b+c) x_{0}, 2(a+c) x_{0}$, and cosine integrals

$$
\operatorname{Ci}(z)=-\int_{z}^{\infty} \frac{\cos t}{t} d t
$$

with $z=2 a x_{0}, 2 b x_{0}, 2 c x_{0}$. Since both $\operatorname{si}(z)$ and $\mathrm{Ci}(z)$ are well-defined functions for $z>0$ [16], we conclude that the integral $F_{4}(a, b, c, d)$ (A.21) is convergent for $\Delta_{4}^{2}=0$. 
We find that eq. (A.22) should be generalized as follows $(a, b, c, d>0)$ :

$$
F_{4}(a, b, c, d)=\left\{\begin{array}{cc}
\frac{1}{\pi^{2} \Delta_{4}} K\left(\frac{\sqrt{a b c d}}{\Delta_{4}}\right), & \Delta_{4}^{2}>a b c d, \\
\frac{1}{\pi^{2} \sqrt{a b c d}} K\left(\frac{\Delta_{4}}{\sqrt{a b c d}}\right), & 0<\Delta_{4}^{2}<a b c d, \\
\frac{1}{2 \pi \sqrt{a b c d}} & \Delta_{4}^{2}=0, \\
0, & \Delta_{4}^{2}<0 .
\end{array}\right.
$$

We took into account that $K(0)=\pi / 2$.

Note that

$$
16 \Delta_{4}^{2}=\left[(c+d)^{2}-(a-b)^{2}\right]\left[(a+b)^{2}-(c-d)^{2}\right] .
$$

So, $\Delta_{4}^{2} \rightarrow \Delta_{3}^{2}$ in the limit $d=0$, and eq. (A.44) (see the first line in its r.h.s) turns into eq. (A.13). Correspondingly, the expression (A.44) transforms into expression (A.2) in the limit $c=d=0$. To demonstrate this, it is enough to put $c+d=\varepsilon \ll 1$, and then repeat arguments used in the derivation of formulas (A.15) - A.20).

Thus, we have shown that (A.2), (A.13) and (A.44) form a self-consistent set of equations in the sense that

$$
\begin{gathered}
\left.F_{4}(a, b, c, d)\right|_{d=0}=F_{3}(a, b, c), \\
\left.F_{4}(a, b, c, d)\right|_{c=d=0}=F_{2}(a, b), \\
\left.F_{3}(a, b, c)\right|_{c=0}=F_{2}(a, b) .
\end{gathered}
$$

Let us stress that this set of equations implies the relations between corresponding analytical expressions for $F_{4}(a, b, c, d)$ (A.44), $F_{3}(a, b, c)$ (A.13), and $F_{2}(a, b)(\mathrm{A} .2) \cdot 7$

Consider now the integral with five Bessel functions $J_{0}(z)$ (as before, $a, b, c, d, e>0)$ :

$$
F_{5}(a, b, c, d, e)=\int_{0}^{\infty} d x x J_{0}(a x) J_{0}(b x) J_{0}(c x) J_{0}(d x) J_{0}(e x) .
$$

\footnotetext{
${ }^{7}$ As for integrals (A.21), (A.12), A.1 themselves, similar relations between them are trivial, taking into account that $J_{0}(0)=1$.
} 
Let us write the identity:

$$
J_{0}(a x) J_{0}(b x)=\int_{0}^{\infty} d y \delta(x-y) J_{0}(a y) J_{0}(b y) .
$$

Using eq. (A.2),

$$
\delta(x-y)=y \int_{0}^{\infty} d t t J_{0}(t x) J_{0}(t y)
$$

we obtain from (A.48):

$$
J_{0}(a x) J_{0}(b x)=\int_{0}^{\infty} d t t J_{0}(t x) \int_{0}^{\infty} d y y J_{0}(a y) J_{0}(b y) J_{0}(t y) .
$$

Thus,

$$
\begin{aligned}
F_{5}(a, b, c, d, e) & =\int_{0}^{\infty} d t t \int_{0}^{\infty} d x x J_{0}(c x) J_{0}(d x) J_{0}(e x) J_{0}(t x) \\
& \times \int_{0}^{\infty} d y y J_{0}(a y) J_{0}(b y) J_{0}(t y)
\end{aligned}
$$

As a result, we come to the following expression:

$$
F_{5}(a, b, c, d, e)=\int_{0}^{\infty} d t t F_{3}(a, b, t) F_{4}(c, d, e, t)
$$

where the functions $F_{3}(a, b, t)$ and $F_{4}(c, d, e, t)$ are defined by formulas (A.13) and (A.44), respectively.

It is possible to get an expression for $F_{5}(a, b, c, d, e)$ which is explicitly symmetric with respect to the parameters $a, b, c, d, e$. First let us note that $e^{2} F_{5}(a, b, c, d, e)=F_{5}(\tilde{a}, \tilde{b}, \tilde{c}, \tilde{d}, 1)$, where 8

$$
\tilde{a}=\frac{a}{e}, \quad \tilde{b}=\frac{b}{e}, \quad \tilde{c}=\frac{c}{e}, \quad \tilde{d}=\frac{d}{e} .
$$

\footnotetext{
${ }^{8}$ Of course, instead of $e$, one can use any of the parameters $a, b, c, d$.
} 
By using equations

$$
\begin{aligned}
& J_{0}(a x) J_{0}(b x)=\int_{0}^{\infty} d t t J_{0}(t x) \int_{0}^{\infty} d y y J_{0}(a y) J_{0}(b y) J_{0}(t y), \\
& J_{0}(c x) J_{0}(d x)=\int_{0}^{\infty} d q q J_{0}(q x) \int_{0}^{\infty} d z z J_{0}(c z) J_{0}(d z) J_{0}(q z),
\end{aligned}
$$

we obtain:

$$
\begin{aligned}
F_{5}(a, b, c, d, e) & =\int_{0}^{\infty} d t t F_{3}(a, b, t) \int_{0}^{\infty} d q q F_{3}(c, d, q) F_{3}(e, t, q) \\
& =\frac{1}{e^{2}} \int_{0}^{\infty} d t t F_{3}(\tilde{a}, \tilde{b}, t) \int_{0}^{\infty} d q q F_{3}(\tilde{c}, \tilde{d}, q) F_{3}(1, t, q)
\end{aligned}
$$

which is symmetric in dimensional parameters $\tilde{a}, \tilde{b}, \tilde{c}, \tilde{d}$.

Note that

$$
\int_{0}^{\infty} d q q F_{3}(c, d, q) F_{3}(e, t, q)=\int_{0}^{\infty} d q q \frac{\theta\left(\Delta_{3}(c, d, q)\right) \theta\left(\Delta_{3}(e, t, q)\right)}{\Delta_{3}(c, d, q) \Delta_{3}(e, t, q)} .
$$

With the help of formula 1.2.37.1. from [19], one can show that after integration in variable $q$, the r.h.s of eq. (A.55) turns into r.h.s of eq. (A.52).

Analogously, we find $(a, b, c, d, e, f>0)$ :

$$
\begin{aligned}
& F_{6}(a, b, c, d, e, f)=\int_{0}^{\infty} d x x J_{0}(a x) J_{0}(b x) J_{0}(c x) J_{0}(d x) J_{0}(e x) J_{0}(f x) \\
& =\int_{0}^{\infty} d t t F_{3}(a, b, t) \int_{0}^{\infty} d q q F_{3}(c, d, q) \int_{0}^{\infty} d p p F_{3}(e, f, p) F_{3}(t, q, p) \\
& =\int_{0}^{\infty} d t t F_{4}(a, b, c, t) F_{4}(d, e, f, t) .
\end{aligned}
$$

The analytic expressions for $F_{3}\left(a_{1}, a_{2}, a_{3}\right)$ and $F_{4}\left(a_{1}, a_{2}, a_{3}, a_{4}\right)$ should be taken from (A.13) and (A.44) 
By doing in the same way, one can express the integrals

$$
F_{n}\left(a_{1}, \ldots, a_{n}\right)=\int_{0}^{\infty} d x x \prod_{k=1}^{n} J_{0}\left(a_{k} x\right)
$$

with $n>6$ and $a_{k}>0, k=1, \ldots, n$ as a $(n-3)$-dimensional integral of algebraic functions. Note that $F_{n}\left(a_{1}, \ldots, a_{n}\right)=0$ if $a_{n}>a_{1}+a_{2}+\ldots a_{n-1}$.

\section{Appendix B}

In this Appendix we study possible restrictions on variables $x_{1}, x_{2}, x_{3}$ in the integral (35) which follow from the inequality (32):

$$
\left[\left(x_{3}+1\right)^{2}-x_{2}^{2}\right]\left[x_{1}^{2}-\left(x_{3}-1\right)^{2}\right]>0 \text {. }
$$

Remember that (see eq. (31))

$$
0 \leqslant x_{1} \leqslant \infty, \quad-x_{1} \leqslant x_{2} \leqslant x_{1}, \quad 0 \leqslant x_{3} \leqslant \infty
$$

If $0 \leqslant x_{3}<1$, we get from (B.1) the following inequalities

$$
\max \left(\left|x_{2}\right|-1,1-x_{1}, 0\right) \leqslant x_{3}<1 \bigcup 0 \leqslant x_{3} \leqslant \min \left(\left|x_{2}\right|-1,1-x_{1}\right) .
$$

Analogously, for $x_{3} \geqslant 1$ eq. (B.1) means that

$$
\max \left(\left|x_{2}\right|-1,1\right) \leqslant x_{3} \leqslant 1+x_{1} \bigcup 1+x_{1} \leqslant x_{3} \leqslant\left|x_{2}\right|-1 .
$$

But since $1+x_{1}>1-\left|x_{2}\right|$, only the first inequality in (B.4) remains in this case:

$$
\max \left(\left|x_{2}\right|-1,1\right) \leqslant x_{3} \leqslant 1+x_{1}
$$


Let $H\left(x_{1}, x_{2}, x_{3}\right)$ be an integrabel function, then

$$
\begin{aligned}
& \int_{0}^{\infty} d x_{1} \int_{-x_{1}}^{x_{1}} d x_{2} \int_{0}^{\infty} d x_{3} \theta\left(\left[\left(x_{3}+1\right)^{2}-x_{2}^{2}\right]\left[x_{1}^{2}-\left(x_{3}-1\right)^{2}\right]\right) H\left(x_{1}, x_{2}, x_{3}\right) \\
& =\left[\int_{0}^{1} d x_{1} \int_{0}^{x_{1}} d x_{2} \int_{1-x_{1}}^{x_{1}+1} d x_{3}+\int_{1}^{2} d x_{1} \int_{0}^{1} d x_{2} \int_{0}^{x_{1}+1} d x_{3}\right. \\
& +\int_{1}^{\infty} d x_{1} \int_{1}^{x_{1}} d x_{2} \int_{\left|x_{2}\right|-1}^{x_{1}+1} d x_{3}+\int_{2}^{\infty} d x_{1} \int_{0}^{x_{1}+1} d x_{2} \int_{0}^{x_{1}+1} d x_{3} \\
& \left.+\int_{2}^{\infty} d x_{1} \int_{1}^{x_{1}} d x_{2} \int_{\left|x_{2}\right|-1}^{\infty} d x_{3}\right]\left[H\left(x_{1}, x_{2}, x_{3}\right)+H\left(x_{1},-x_{2}, x_{3}\right)\right] .
\end{aligned}
$$

\section{References}

[1] G. Molière, Theorie der Streuung schneller geladener Teilchen. I. Einzelstreuung am abgeschirmten Coulomb-Feld, Z. Naturforsch, 2a (1947) 133; Theorie der Streuung schneller geladener Teilchen. II. Mehrfachund Vielfachstreuung, Z. Naturforsch, 3a (1948) 78.

[2] R. J. Glauber, in Lectures in Theoretical Physics, ed. W.E. Brittin and L.G. Dunham, New York, vol. 1, 1959, p. 315.

[3] R.G. Newton, Scattering Theory of Waves and Particles, McGraw-Hill Book Company, New York, 1966.

[4] H. Cheng and T.T. Wu, High-energy elastic scattering in quantum electrodynamics, Phys. Rev. Lett., 22 (1969) 666; Impact factor and exponentiation in high-energy scattering processes, Phys. Rev., 186 (1969) 1611.

[5] A.A. Logunov, A.N. Tavkhe1idze, Quasioptical approach in quantum field theory, Nuovo Cim., 29 (1963) 380.

[6] V.R. Garsevanishvili, V.A. Matveev, L.A. S1epchenko and A.N. Tavkhe1idze, Eikonal approximation in quantum-field theory, Phys. Lett., 29B (1969) 191. 
[7] V.A. Petrov, High-energy implications of extended unitarity, in Proceedings of the 7th Rencontres de Blois, 7th Blois Workshop on Elastic and Diffractive Scattering, Blois, France, June 20-24, 1995, pp. 139-143.

[8] A.V. Kisselev and V.A. Petrov, Gravireggeons in extra dimensions and interaction of ultra-high energy cosmic neutrinos with nucleons, Eur. Phys. J. C, 36 (2004) 103; Eikonal amplitude in the gravireggeon model at superplanckian energies, Eur. Phys. J. C, 37 (2004) 241.

[9] J.D. Jackson, Classical Electrodynamics, 3rd edition, J.Wiley \& Sons, Inc., US, 1998.

[10] I.S. Gradshteyn and I.M. Ryzhik, Table of Integrals, Series, and Products, 7th edition, Academic Press, New York, 2007.

[11] G.N. Watson, A Treatise on the Theory of Bessel Functions, 2nd edition, Cambridge University Press, 1995.

[12] I.M. Gel'fand and G.E. Shilov. Generalized Functions. Volume I: Properties and Operations. Academic Press, New York, 1964.

[13] M. Abramowitz and I.A. Stegun, eds., Handbook of Mathematical Functions with formulas, Graphs and Mathematical Tabales, NBS Applied Mathematics Series 55, National Bureau of Standards, 10th edition, Washington, DC, 1972.

[14] A.P. Prudnikov, Yu.A. Brychkov and O.I. Marichev, Integrals and Series, Vol. 2: Special Functions, Gordon \& Breach Sci. Publ., New York, 1986.

[15] Higher Transcendental Functions. Vol. 3. By the staff of the Bateman manuscript project (A. Erdélyi, Editor; W. Magnus, F. Oberhettinger, F.G. Tricomi, Associates), McGraw-Hill Book Company, New York, 1955.

[16] Higher Transcendental Functions. Vol. 2. By the staff of the Bateman manuscript project (A. Erdélyi, Editor; W. Magnus, F. Oberhettinger, F.G. Tricomi, Associates), McGraw-Hill Book Company, New York, 1953. 
[17] M.L. Glasser and E. Montaldi, Some integrals Involving Bessel Functions, arXiv:math/9307213, 1993.

[18] Higher Transcendental Functions. Vol. 1. By the staff of the Bateman manuscript project (A. Erdélyi, Editor; W. Magnus, F. Oberhettinger, F.G. Tricomi, Associates), McGraw-Hill Book Company, New York, 1953.

[19] A.P. Prudnikov, Yu.A. Brychkov and O.I. Marichev, Integrals and Series, Vol. 1: Elementary Functions, Gordon \& Breach Sci. Publ., New York, 1986. 